\title{
Computation of azimuthal waves and their stability in thermal convection in rotating spherical shells with application to the study of a double-Hopf bifurcation
}

\author{
J. Sánchez, ${ }^{*}$ F. Garcia, and M. Net \\ Departament de Física Aplicada, Universitat Politècnica de Catalunya, Jordi Girona Salgado 1-3, Campus Nord, \\ Mòdul B4, 08034 Barcelona, Spain
}

(Received 14 January 2013; published 22 March 2013)

\begin{abstract}
A methodology to compute azimuthal waves, appearing in thermal convection of a pure fluid contained in a rotating spherical shell, and to study their stability is presented. It is based on continuation, Newton-Krylov, and Arnoldi methods. An application to the study of a double-Hopf bifurcation of the basic state is shown for Ekman and Prandtl numbers $E=10^{-4}$ and $\sigma=0.1$, respectively, radius ratios $\eta \in[0.32,0.35]$, Rayleigh numbers $R \in\left[1.8 \times 10^{5}, 6 \times 10^{5}\right]$, and nonslip and perfectly conducting boundary conditions. The knowledge of the bifurcation diagrams, including the unstable solutions, allows one to understand the coexistence of stable thermal Rossby waves of different azimuthal wave numbers at some parameter regions, and the origin of some new intermittent solutions found, as trajectories close to heteroclinic chains. Moreover, the structure of the eigenfunctions at the secondary bifurcations explains the existence of the amplitude and shape modulated waves.
\end{abstract}

DOI: 10.1103/PhysRevE.87.033014

PACS number(s): 47.20.Bp, 47.55.P-

\section{INTRODUCTION}

Thermal convection in rotating spherical fluid shells subject to a radial gravity field is fundamental in geophysical and astrophysical fluid dynamics. Its study allows one to estimate the transport of energy in the interior of planets and stars, determine their internal structure and its influence on the patterns observed in the upper atmospheres of giant planets, provide models to fit to experimental observations of the drifting of these internal patterns, understand the generation of magnetic fields if coupled with the induction equation, etc. From a mathematical point of view the difficulties of the spherical geometry in the rotating case make the attempts of analytical treatment extremely challenging, requiring very careful sequences of approximations to preserve the relevant features. The tools used to study the problem have been those of hydrodynamic stability theory, to obtain the critical value of the parameters, and the structure of the solutions at the onset of convection by using analytical, semianalytical [1-6], or numerical methods [7-10], and time evolution codes to obtain the nonlinear solutions at low [11-13] or high supercritical Rayleigh numbers [14-17], to mention just a few of all the available references.

The computation of the azimuthal waves by Newton's methods and the study of their stability by using direct linear algebra methods were performed, for instance, in $[18,19]$ and more recently in [20], where curves of the secondary transitions in the Taylor-Rayleigh numbers plane were presented for $\sigma=1$, and $E$ reaching a minimum value of $4 \times 10^{-3}$. These methods allow more detailed calculations in a wider range of parameters than just using time integrations. Moreover, unstable objects can be found which can be relevant in the global dynamics. In order to be able to decrease the Ekman number and, consequently [9], increase the Rayleigh number it is necessary to use iterative linear algebra methods to cope with the required size of the systems. Some preliminary results using these techniques in the context of continuation methods

*Juan.J.Sanchez@upc.edu were presented in [21]. In this paper the computational details are given, and an application to the computation of the waves close to a double-Hopf bifurcation is shown.

Computations based on continuation of steady states or traveling waves of high-dimensional systems are nowadays common. Those of periodic orbits of nontrivial time dependence [22-26] and even tori [27] or other invariant objects [28] are now starting to appear, and will surely contribute to a better understanding of more complex behaviors and the transition to turbulence. In the problem studied here, a first step is having efficient tools to reach the bifurcation point of the azimuthal waves and to find the corresponding eigenfunctions, in the case of the high dimensions needed at low Ekman numbers. For instance, they allow one to find different branches of stable solutions that could eventually give rise to different chaotic regimes for the same values of the parameters.

The quasiperiodic patterns of convection, i.e., the amplitude and the shape modulated waves, bifurcated from the thermal Rossby waves in internally heated, self-gravitating rotating spherical fluid shells at moderate and large $\sigma$ are well described in $[16,19]$. By means of the stability analysis of the pure waves, without enforcing any particular form of the perturbation, it will be shown that for differential heating and moderate $\sigma$, unstable shape modulated waves (which cannot be observed by just time evolutions) appear at an azimuthal symmetry breaking bifurcation on the second branch (initially unstable) of solutions emanating from the conduction state. This behavior is explained by the unfolding of the double-Hopf bifurcation. Moreover, the secondary instability on the first branch does not break the azimuthal symmetry. Then stable shape modulated waves exist only after tertiary bifurcations.

According to the nonslip boundary conditions employed, the parameters selected for the computations correspond to the radius ratio of the present day Earth's outer core $\eta \approx 0.35$, its estimated Prandtl number $\sigma=0.1$ [29,30], and an Ekman number at the beginning of the asymptotic limit of low $E$ for the onset of convection [31]. Nowadays it is impossible to reach either experimentally or numerically the real $E \approx 10^{-15}$ estimated for the Earth's outer core, although with the method proposed here calculations with 
$E \approx 10^{-7}$ would be possible for geophysical or astrophysical applications. Notice that this last $E$ with stress-free or mixed boundary conditions approximates well that needed in some astrophysical simulations, for instance to model the upper atmosphere of Jupiter.

The paper is organized as follows. Section II describes the problem and the initial equations to be solved, Sec. III accounts for the spatial discretization in spherical harmonics and the equations for the amplitudes of the expansions. In Secs. IV and V the methods to follow the branches of waves bifurcated from the conduction state and to study their stability are explained. The results obtained are shown in Sec. VI. Finally, some conclusions and possible lines of future work are summarized in Sec. VII.

\section{THERMAL CONVECTION IN ROTATING SPHERICAL SHELLS}

Consider a spherical shell of inner and outer radii $r_{i}$ and $r_{o}$, respectively, rotating about a fixed axis at angular velocity $\Omega \mathbf{k}$ (k being the unit vector defining the $z$ direction), filled with a pure fluid subject to a difference of temperature $\Delta T=$ $T_{i}-T_{o}>0$, and to a radial gravity $\mathbf{g}=-\gamma \mathbf{r}$, with $\gamma>0$ and $\mathbf{r}=(x, y, z)$ (corresponding to the field inside a sphere of uniform density). We also assume that the centrifugal force can be neglected when it is compared with gravity, i.e., $\Omega^{2} / \gamma \ll$ 1. This is the case in many geophysical and astrophysical problems which are the origin of this problem.

The equations governing the dynamics of the fluid in the Boussinesq approximation are written in spherical coordinates $(r, \theta, \varphi), \theta$ and $\varphi$ being the colatitude and longitude, respectively, in the rotating frame of reference of the spheres, and in nondimensional form by taking as units of length, time, temperature, and pressure $d, d^{2} / \nu, v^{2} / \gamma \alpha d^{4}$, and $\rho_{0} v^{2} / d$, respectively, where $\rho_{0}$ is the density at a reference temperature, $d=r_{o}-r_{i}$, and $v$ is the kinematic viscosity. They are

$$
\begin{aligned}
\partial_{t} \mathbf{v}+(\mathbf{v} \cdot \nabla) \mathbf{v}+2 E^{-1} \mathbf{k} \times \mathbf{v} & =-\nabla \Pi+\nabla^{2} \mathbf{v}+\Theta \mathbf{r} \\
\nabla \cdot \mathbf{v} & =0 \\
\sigma\left(\partial_{t} \Theta+\mathbf{v} \cdot \nabla \Theta\right) & =\nabla^{2} \Theta+R \eta(1-\eta)^{-2} r^{-3} \mathbf{r} \cdot \mathbf{v}
\end{aligned}
$$

where $\Theta=T-T_{c}$, is the temperature perturbation from the conduction state $\mathbf{v}=0$ and $T_{c}(r)=T_{0}+R \eta / \sigma(1-\eta)^{2} r$, which is always a solution in this approximation, if constant temperature at the boundaries is considered. The modified pressure $\Pi$ contains the pressure and additional terms coming from the dependence of the density with the temperature in the buoyancy term. In addition, nonslip boundary conditions are enforced.

The nondimensional parameters of the problem, which appear in the equations, are the radius ratio, and the Prandtl, Ekman, and Rayleigh numbers defined as

$$
\eta=\frac{r_{i}}{r_{o}}, \quad \sigma=\frac{\nu}{\kappa}, \quad E=\frac{v}{\Omega d^{2}}, \quad \text { and } \quad R=\frac{\gamma \alpha \Delta T d^{4}}{\kappa \nu},
$$

respectively, with $\kappa$ the thermal diffusivity. The dependence of the solutions with the Rayleigh number is studied for $0.32 \leqslant$ $\eta \leqslant 0.35,1.8 \times 10^{5} \leqslant R \leqslant 6 \times 10^{5}, \sigma=0.1$, and $E=10^{-4}$. For this $E$ the linear stability analysis of the conduction state in the asymptotic limit of low Ekman numbers is valid, and the Taylor-Proudman theorem applies.

The symmetry group of the system when $\Omega \neq 0$ is $\mathrm{SO}(2) \times$ $\mathbb{Z}_{2}$ generated by the rotations about the $z$ axis, and the reflection with respect to the equatorial plane. When the conduction state becomes unstable at a Hopf bifurcation, which breaks the rotational invariance of the conduction state, it gives rise to traveling azimuthal waves [32].

In order to satisfy the zero-divergence condition, to eliminate the pressure, and to reduce the number of equations, two scalar potentials (toroidal and poloidal) for the velocity field are introduced [1], i.e.,

$$
\mathbf{v}=\nabla \times(\Psi \mathbf{r})+\nabla \times \nabla \times(\Phi \mathbf{r}) .
$$

The equations for the potentials are obtained by applying the operators $\mathbf{r} \cdot \nabla \times$ and $\mathbf{r} \cdot \nabla \times \nabla \times$ to the momentum equation. They will be written later once discretized.

\section{EXPANSION IN SPHERICAL HARMONICS}

To discretize the equations, the velocity potentials and the perturbation of the temperature from the conduction state are expanded in spherical harmonic series, truncated at degree (index $l$ ) and order (index $m$ ) $L$ as

$$
(\Psi, \Phi, \Theta)(t, r, \theta, \varphi)=\sum_{\substack{l=0, \ldots, L \\ m=-l, \ldots, l \\ m \\=\dot{m}_{d}}}\left(\Psi_{l}^{m}, \Phi_{l}^{m}, \Theta_{l}^{m}\right)(t, r) Y_{l}^{m}(\theta, \varphi)
$$

(a triangular truncation). The spherical harmonics are normalized as

$$
Y_{l}^{m}(\theta, \varphi)=\sqrt{\frac{2 l+1}{2} \frac{(l-m) !}{(l+m) !}} P_{l}^{m}(\cos \theta) e^{i m \varphi},
$$

and $\Psi_{l}^{-m}=\overline{\Psi_{l}^{m}}, \Phi_{l}^{-m}=\overline{\Phi_{l}^{m}}$, and $\Theta_{l}^{-m}=\overline{\Theta_{l}^{m}}$. Moreover, to have the two potentials completely determined we can choose $\Psi_{0}^{0}=\Phi_{0}^{0}=0$.

Since the waves emerging at the primary bifurcation have an integer azimuthal wave number $m_{d}$, which is usually $m_{d}>$ 1 , the expansion can include only the orders $m$ multiple of a selected wave number $m_{d}$ to reduce the dimension of the systems to be solved. If $m_{d}=1$ we include all the spherical harmonics. Since the waves are in most cases symmetric with respect the equatorial reflection, it would be possible to reduce even more the size by considering the expansion in symmetric spherical harmonics for $\Phi$ and $\Theta$, and antisymmetric for $\Psi$.

In the radial coordinate we use collocation on a GaussLobatto mesh of $n_{r}+1$ points, including the boundary condition into the discretized operators. By using collocation all the radial operators are substituted by matrices. Each time the evaluation of an operator is required, all similar computations are grouped to call efficient implementations (ATLAS, GOTOBLAS, etc.) of the matrix-matrix product subroutine DGEMM of BLAS $[33,34]$. The ordering of the variables was chosen to implement this idea and the application of FFTs using FFTW [35].

The unknowns are then the values of the amplitudes $\Psi_{l}^{m}, \Phi_{l}^{m}$, and $\Theta_{l}^{m}$ at the inner points of the radial mesh. In the calculations shown $30 \leqslant n_{r} \leqslant 50$, and $70 \leqslant L \leqslant$ 90 depending on the preferred $m_{d}$. The total dimension 
TABLE I. Variation with the truncation parameters of the dimensions of the system to find the waves $\left(n_{\text {wave }}\right)$ and to study their stability $\left(n_{\text {stab }}\right)$, and the critical Rayleigh number $\left(R_{c}\right)$, the drifting frequency $(\omega)$, and the imaginary part of the crossing eigenvalue $[\operatorname{Im}(\lambda)]$, at three bifurcations along the curves of waves for $\eta=0.35$. The first two blocks correspond to the two limits of the stable portion of the $m=5$ branch, and the third to the secondary bifurcation of the $m=6$. They bifurcate from the conduction state at $R_{c}^{0}=191176.44$ $(m=5)$, and $R_{c}^{0}=18606.91(m=6)$.

\begin{tabular}{cccrcccc}
\hline \hline$n_{r}$ & $m_{d}$ & $L / m_{d}$ & $n_{\text {wave }}$ & $n_{\text {stab }}$ & $R_{c}$ & $\omega$ & $\operatorname{Im}(\lambda)$ \\
\hline 30 & 5 & 14 & 87725 & 438509 & 204342.8 & 92.7706 & 79.7529 \\
35 & 5 & 14 & 102850 & 514114 & 204344.6 & 92.7701 & 79.7523 \\
40 & 5 & 14 & 117975 & 589719 & 204344.5 & 92.7701 & 79.7521 \\
50 & 5 & 14 & 148225 & 740929 & 204344.5 & 92.7701 & 79.7523 \\
40 & 5 & 18 & 193791 & 968799 & 204344.7 & 92.7700 & 79.7523 \\
35 & 5 & 14 & 102850 & 102850 & 273851.8 & 77.3355 & 178.120 \\
40 & 5 & 14 & 117975 & 117975 & 273850.8 & 77.3357 & 178.120 \\
50 & 5 & 14 & 148225 & 148225 & 273851.5 & 77.3356 & 178.120 \\
40 & 5 & 18 & 193791 & 193791 & 273852.5 & 77.3354 & 178.120 \\
40 & 5 & 24 & 342615 & 342615 & 273852.1 & 77.3355 & 178.121 \\
30 & 6 & 14 & 104777 & 104777 & 258438.8 & 62.7038 & 172.312 \\
35 & 6 & 14 & 122842 & 122842 & 258440.6 & 62.7032 & 172.314 \\
40 & 6 & 14 & 140907 & 140907 & 258441.7 & 62.7031 & 172.315 \\
50 & 6 & 14 & 177037 & 177037 & 258441.0 & 62.7032 & 172.315 \\
40 & 6 & 18 & 291109 & 291109 & 258441.0 & 62.7032 & 172.314 \\
\hline \hline
\end{tabular}

$n=\left(n_{r}-1\right)\left[\left(3 L^{2}+6 L\right) / m_{d}+1\right]$ is in the range $87725 \leqslant$ $n \leqslant 968799$ depending on $n_{r}, L$, and $m_{d}$ (more details will be given in Table I below).

The equations for the amplitudes are

$$
\begin{aligned}
\partial_{t} \Psi_{l}^{m}= & \mathcal{D}_{l} \Psi_{l}^{m}+\frac{1}{l(l+1)}\left\{2 E^{-1}\left(i m \Psi_{l}^{m}-[Q \Phi]_{l}^{m}\right)\right. \\
- & {\left.[\mathbf{r} \cdot \nabla \times(\boldsymbol{\omega} \times \mathbf{v})]_{l}^{m}\right\} } \\
\partial_{t} \mathcal{D}_{l} \Phi_{l}^{m}= & \mathcal{D}_{l}^{2} \Phi_{l}^{m}-\Theta_{l}^{m}+\frac{1}{l(l+1)}\left\{2 E ^ { - 1 } \left(i m \mathcal{D}_{l} \Phi_{l}^{m}\right.\right. \\
& \left.\left.+[Q \Psi]_{l}^{m}\right)+[\mathbf{r} \cdot \nabla \times \nabla \times(\boldsymbol{\omega} \times \mathbf{v})]_{l}^{m}\right\}, \\
\partial_{t} \Theta_{l}^{m}= & \sigma^{-1} \mathcal{D}_{l} \Theta_{l}^{m}+\sigma^{-1} l(l+1) R \eta(1-\eta)^{-2} r^{-3} \Phi_{l}^{m} \\
& -[\mathbf{v} \cdot \nabla \Theta]_{l}^{m},
\end{aligned}
$$

for $0 \leqslant l \leqslant L$, and $0 \leqslant m=\dot{m}_{d} \leqslant l$ (if $l=0$ and $m=0$ only the equation for $\Theta_{0}^{0}$ is present). In the first two equations $\boldsymbol{\omega}=\boldsymbol{\nabla} \times \mathbf{v}$ is the vorticity, $\mathcal{D}_{l}=\partial_{r r}^{2}+(2 / r) \partial_{r}-l(l+1) / r^{2}$, and the operator $Q$ is defined as

$[Q f]_{l}^{m}=-l(l+2) c_{l+1}^{m} D_{l+2}^{+} f_{l+1}^{m}-(l-1)(l+1) c_{l}^{m} D_{1-l}^{+} f_{l-1}^{m}$, with $D_{l}^{+} f=\partial_{r} f+l f / r$, and $c_{l}^{m}=\left[\left(l^{2}-m^{2}\right) /\left(4 l^{2}-1\right)\right]^{1 / 2}$.

On the boundaries $r_{i}=\eta /(1-\eta)$ and $r_{o}=1 /(1-\eta)$ the nonslip boundary conditions $\operatorname{read} \Phi_{l}^{m}=\partial_{r} \Phi_{l}^{m}=\Psi_{l}^{m}=0$. These are more demanding than the stress-free from a computational point of view because the Ekman boundary layers must be solved. The perfectly conducting conditions in terms of the perturbation of the temperature are $\Theta_{l}^{m}=0$. For the evaluation of the nonlinear terms in Eqs. (2)-(4) the formulas given in [7] were used, and their computation was de-aliased (see [36]).
We define the inner product used below to write the equations giving rise to the waves. If

$$
f(r, \theta, \varphi)=\sum_{m=-L}^{L} \sum_{l=|m|}^{L} f_{l}^{m}(r) Y_{l}^{m}(\theta, \varphi)
$$

with $f_{l}^{-m}=\overline{f_{l}^{m}}$, and the same for $g$, then

$$
\langle f, g\rangle=\frac{1}{2 \pi} \int_{r_{i}}^{r_{o}} d r \int_{0}^{2 \pi} d \varphi \int_{0}^{\pi} \bar{f}(r, \theta, \varphi) g(r, \theta, \varphi) \sin \theta d \theta .
$$

Therefore

$$
\langle f, g\rangle=\sum_{m=-L}^{L} \sum_{l=|m|}^{L} \int_{r_{i}}^{r_{o}} \overline{f_{l}^{m}}(r) g_{l}^{m}(r) d r
$$

where we have used that with the normalization of Eq. (1) the spherical harmonics verify the orthogonality relation

$$
\frac{1}{2 \pi} \int_{0}^{2 \pi} d \varphi \int_{0}^{\pi} \overline{Y_{l_{1}}^{m_{1}}}(\theta, \varphi) Y_{l_{2}}^{m_{2}}(\theta, \varphi) \sin \theta d \theta=\delta_{l_{1}, l_{2}} \delta_{m_{1}, m_{2}} .
$$

Finally, if $u=(\Psi, \Phi, \Theta)$ and $u^{\prime}=\left(\Psi^{\prime}, \Phi^{\prime}, \Theta^{\prime}\right)$, we define $\left\langle u, u^{\prime}\right\rangle \equiv\left\langle\Psi, \Psi^{\prime}\right\rangle+\left\langle\Phi, \Phi^{\prime}\right\rangle+\left\langle\Theta, \Theta^{\prime}\right\rangle$, and $\|u\|_{L^{2}}=\langle u, u\rangle^{1 / 2}$. When the solutions are also discretized in the radius, the integrals in Eq. (6) are approximated by Clenshaw-Curtis quadrature formulas to plot $\|u\|_{L^{2}}$ against the continuation parameter. However, when the scalar product is used to add a phase condition on the fully discretized system, it is substituted by the simpler formula

$$
\langle f, g\rangle=\sum_{m=-L}^{L} \sum_{l=|m|}^{L} \sum_{i=1}^{n_{r}-1} \overline{f_{l}^{m}}\left(r_{i}\right) g_{l}^{m}\left(r_{i}\right),
$$

where the $r_{i}, i=1, \ldots, n_{r}-1$ are the inner collocation points.

\section{CONTINUATION OF THE WAVES}

The system (2)-(4) will be written in compact form as

$$
L_{0} \partial_{t} u=L u+B(u, u),
$$

where now $u$ is the vector containing the values of the coefficients of the expansions in spherical harmonics at the mesh of collocation points in the radius. The block diagonal linear operator $L_{0}$ acts as the identity on $\Psi_{l}^{m}$ and $\Theta_{l}^{m}$ and as $\mathcal{D}_{l}$ on $\Phi_{l}^{m}$, and $L$ and $B$ are, respectively, linear and bilinear operators, $L$ depending on all the parameters of the problem; in particular on the Rayleigh number $p=R$, which is the continuation parameter used here. The dependence $L=L(p)$ will be written explicitly when required.

The continuation with respect to the parameters $E$ and $\sigma$ is straightforward. When $\eta$ is the continuation parameter, even the domain of computation depends on it, and it is necessary to recompute almost everything each time it is changed. The domain should be parametrized, and therefore all the radial derivatives would depend on $\eta$. Moreover $L$ is not linear in $\eta$, and the quadratical dependence of the nonlinear terms for fixed $\eta$ is lost. This continuation could be probably done, but would surely be quite difficult.

At critical values of $p=p_{c}$, the conduction state $u=0$ becomes unstable, and branches of azimuthal traveling waves start there at Hopf bifurcations. Then, at these values of $p$, 
there are vectors $v_{c}$ and frequencies $\omega_{c}$ such that $i \omega_{c} L_{0} v_{c}=$ $L\left(p_{c}\right) v_{c}$. These waves with temporal dependence $u(t, r, \theta, \varphi)=$ $\tilde{u}(r, \theta, \varphi-\omega t)$, satisfy $\omega L_{0} \partial_{\tilde{\varphi}} \tilde{u}+L \tilde{u}+B(\tilde{u}, \tilde{u})=0$, with $\tilde{\varphi}=\varphi-\omega t$ or, by deleting the tildes,

$$
F(u, \omega, p) \equiv \omega L_{0} \partial_{\varphi} u+L(p) u+B(u, u)=0 .
$$

This equation must be supplemented with another which fixes the phase of the wave. We use the condition

$$
G(u)=\left\langle u, \partial_{\varphi} u_{c}\right\rangle=0,
$$

where $u_{c}$ is a reference solution (the eigenvector, $u_{c}=v_{c}$, at $p=p_{c}$, or a previously computed solution). It is a necessary condition for $\left\|u-u_{c}\right\|_{2}^{2}$ to be minimal with respect to the phase. Let

$$
H(\alpha)=(1 / 2)\left\|u(r, \theta, \varphi+\alpha)-u_{c}(r, \theta, \varphi)\right\|_{2}^{2},
$$

and for convenience $u(\alpha)=u(r, \theta, \varphi+\alpha)$ then

$$
\begin{aligned}
H(\alpha) & =(1 / 2)\left\|u(\alpha)-u_{c}\right\|_{2}^{2} \\
& =\left(\langle u(\alpha), u(\alpha)\rangle+\left\langle u_{c}, u_{c}\right\rangle\right) / 2-\left\langle u(\alpha), u_{c}\right\rangle,
\end{aligned}
$$

and

$$
\frac{d H}{d \alpha}(\alpha)=-\left\langle\partial_{\varphi} u(\alpha), u_{c}\right\rangle=\left\langle u(\alpha), \partial_{\varphi} u_{c}\right\rangle,
$$

because $\left\langle u_{c}, u_{c}\right\rangle$ is a constant, and $\langle u(\alpha), u(\alpha)\rangle$ is also constant if $u$ is an azimuthal wave. The second identity holds because, if $f$ and $g$ are $2 \pi$-periodic functions of $\varphi$, then

$$
\int_{0}^{2 \pi} \partial_{\varphi} f g d \varphi+\int_{0}^{2 \pi} f \partial_{\varphi} g d \varphi=\int_{0}^{2 \pi} \partial_{\varphi}(f g) d \varphi=0 .
$$

Moreover, if $H(\alpha)$ has a minimum at $\alpha_{0}$ then

$$
\begin{aligned}
0 & <\frac{d^{2} H}{d \alpha^{2}}\left(\alpha_{0}\right)=\frac{d G}{d \alpha}\left(u\left(\alpha_{0}\right)\right)=\partial_{u} G\left(u\left(\alpha_{0}\right)\right) \partial_{\varphi} u\left(\alpha_{0}\right) \\
& =\left\langle\partial_{\varphi} u\left(\alpha_{0}\right), \partial_{\varphi} u_{c}\right\rangle .
\end{aligned}
$$

This result will be used later.

To study the dependence of the waves on $p$, we use a pseudoarclength continuation method which allows us to obtain the curves of solutions $(u(s), \omega(s), p(s)), s$ being its arclength, by adding an equation

$N(u, \omega, p) \equiv w_{u}^{\top}\left(u-u^{0}\right)+w_{\omega}\left(\omega-\omega^{0}\right)+w_{p}\left(p-p^{0}\right)=0$, where $\left(u^{0}, \omega^{0}, p^{0}\right)$ and $\left(w_{u}, w_{\omega}, w_{p}\right)$ are predictions of a point and the tangent to the curve of solutions, respectively, obtained by extrapolation from the previous points as depicted in Fig. 1. This parametrization allows one to pass possible folds. Some of them have been found in the examples of Sec. VI.

The system which determines a unique solution, $(u, \omega, p) \in$ $\mathbb{R}^{n+2}$, is then

$$
F(u, \omega, p)=0, \quad G(u)=0, \quad N(u, \omega, p)=0 .
$$

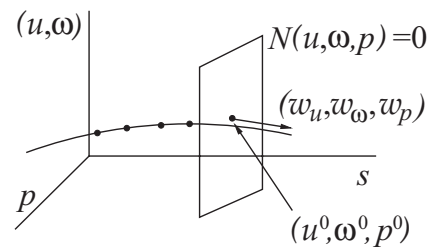

FIG. 1. Continuation procedure.
Newton-Krylov methods are used to solve it. For the linear systems we use GMRES [37], which requires computing the action of the matrix and a suitable preconditioner. The Jacobian of the above system is

$$
\left(\begin{array}{ccc}
\partial_{u} F & \partial_{\omega} F & \partial_{p} F \\
\partial_{u} G & 0 & 0 \\
w_{u}^{\top} & w_{\omega} & w_{p}
\end{array}\right) .
$$

The action of the first block of rows of the Jacobian $\left(\partial_{u} F, \partial_{\omega} F, \partial_{p} F\right)(u, \omega, p)$ on $(v, \zeta, \mu)$ is

$$
\omega L_{0} \partial_{\varphi} v+L(p) v+\zeta L_{0} \partial_{\varphi} u+\mu L^{(2)} u+B(u, v)+B(v, u),
$$

due to the dependence of $L$ on $p$, which has the form $L(p)=$ $L^{(1)}+p L^{(2)}$ for $p=R, \sigma^{-1}$, or $2 E^{-1}$. The action of $\partial_{u} G(u)$ on $v$ is $\partial_{u} G(u) v=\left\langle v, \partial_{\varphi} u_{c}\right\rangle$.

The Jacobian $\partial_{u} F$ is singular on the solutions of $F(u, \omega, p)=0$, because if $u(r, \theta, \varphi)$ is a solution then so also $u(r, \theta, \varphi+\alpha)$ for any phase $\alpha$. Then $F(u(r, \theta, \varphi+\alpha), \omega, p)=0$ for any $\alpha$, and by taking the derivative with respect to $\alpha$, $\partial_{u} F \partial_{\varphi} u=0$, i.e., $\partial_{\varphi} u$ is a zero eigenvector of $\partial_{u} F$ (provided $u$ depends on $\varphi$, i.e., the solution is not axisymmetric). However, there is generically no zero eigenvector of the Jacobian

$$
\left(\begin{array}{cc}
\partial_{u} F & \partial_{\omega} F \\
\partial_{u} G & 0
\end{array}\right)
$$

of the form $\left(\partial_{\varphi} u, \beta\right)^{\top}$. If this were the case, $\beta \partial_{\omega} F$ and $\partial_{u} G \partial_{\varphi} u$ would be zero, but we have seen that if $H(\alpha)$ has a nondegenerate minimum, $\partial_{u} G \partial_{\varphi} u>0$. Moreover, since $\partial_{\omega} F=L_{0} \partial_{\varphi} u$, if $u$ depends on $\varphi$ and $L_{0}$ is nonsingular, the last column of the Jacobian does not generically vanish. This Jacobian can only be singular at bifurcation points.

As preconditioner we have used matrices of the form

$$
\left(\begin{array}{ccc}
\omega_{\text {prec }} L_{0} \partial_{\varphi}+L_{\text {prec }} & 0 & 0 \\
0 & 1 & 0 \\
0 & 0 & 1
\end{array}\right),
$$

with $L_{\text {prec }}=L\left(p_{\text {prec }}\right)$ and $\omega_{\text {prec }}$ being the operator $L$ and the frequency of the waves at a previous step. Since $L$ has block-tridiagonal structure, due to the operator $Q$, which couples the spherical harmonics coefficients of degrees $l-1$, $l$, and $l+1$, it is possible to solve this latter system efficiently by an adapted LU decomposition. This has proved to be a very efficient preconditioner for the calculations presented here because, in the region of interest, the solutions are relatively close to the conduction state, for which the first block of rows of the preconditioner is exact if $p_{\text {prec }}=p$. It is possible to keep the preconditioner fixed for several steps, but we have seen that, in order to compute the branches of waves in the most efficient way, it is better to update it frequently. Since the continuation code includes a control of the arclength stepsize, which increases from the beginning of the branch to the final point, we decided to update the preconditioner after each successful computation of a new solution, without increasing significantly the total computation time of a branch of solutions. 

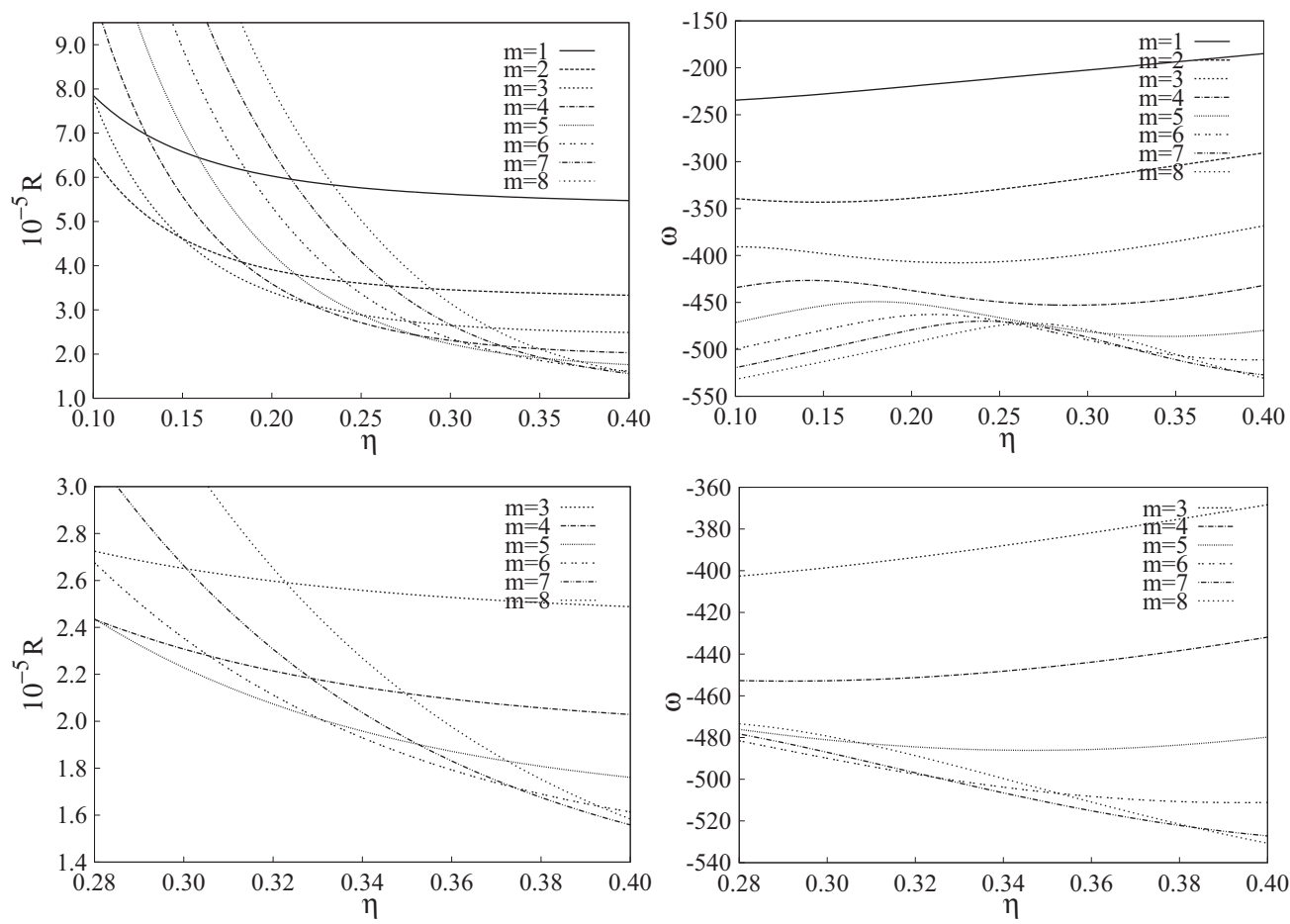

FIG. 2. Critical Rayleigh number and frequencies versus $\eta$ for $m=1, \ldots, 8, \sigma=0.1$, and $E=10^{-4}$, and details close to the region studied.

\section{STABILITY OF THE WAVES}

Suppose a wave of the form $u(r, \theta, \varphi-\omega t)$ has been found. To study its stability, the first option is to apply Floquet theory which implies integrating the variational equation

$$
L_{0} \partial_{t} v=L v+B(u(t), v)+B(v, u(t)),
$$

where we have written $u(t)=u(r, \theta, \varphi-\omega t)$. If $\chi(t, v)$ is its solution with initial condition $v$ at $t=0$, the eigenvalues of the map $v \longrightarrow \chi(\tau, v)$ must be computed, $\tau$ being the period of the wave. The +1 eigenvalue, corresponding to the eigenfunction $v_{1} \equiv L_{0}^{-1}(L u+B(u, u))$ at $t=0$, can be deflated by computing the eigenvalues of the map,

$$
v \longrightarrow \chi(t, v)-\left\langle\chi(t, v), v_{1}\right\rangle v_{1} .
$$

This method is very robust but expensive because it requires time integrations, therefore it was only used to test the second method described next, and to check that the bifurcation points detected were the same for both.

The second method consists in studying the stability of $u$ as a fixed point of a vector field. Consider a perturbation $v(t, r, \theta, \tilde{\varphi})$ of $u(r, \theta, \tilde{\varphi})$, and

$$
\hat{u}(t, r, \theta, \varphi)=u(r, \theta, \tilde{\varphi})+v(t, r, \theta, \tilde{\varphi}) .
$$

After substituting it into the initial system (8), and neglecting the second order terms, the linearized equation

$$
L_{0} \partial_{t} v=L v+\omega L_{0} \partial_{\varphi} v+B(u, v)+B(v, u)
$$

is obtained. Then the eigenvalue problem

$$
\lambda L_{0} v=L v+\omega L_{0} \partial_{\varphi} v+B(u, v)+B(v, u),
$$

or, since $L_{0}$ is invertible, $\lambda v=\mathcal{L} v$ with

$$
\mathcal{L} v \equiv L_{0}^{-1}\left[L v+\omega L_{0} \partial_{\varphi} v+B(u, v)+B(v, u)\right]
$$

must be solved. Matrix transformations must be used (real shift invert, Cayley, shift invert with complex shift and real arithmetic, double complex shift, etc.) to extract the right-most eigenvalues [38,39]. The transformations change the eigenvalues but not the eigenvectors. Two of them were implemented. For the real shift invert with shift $\sigma_{s i}$, the transformed eigenvalue problem, and the relation between the eigenvalues are

$$
\left(\mathcal{L}-\sigma_{s i} \mathcal{I}\right)^{-1} v=\mu_{s i} v, \quad \mu_{s i}=1 /\left(\lambda-\sigma_{s i}\right)
$$

For Cayley transformations they are

$$
\left(\mathcal{L}-\sigma_{c} \mathcal{I}\right)^{-1}\left(\mathcal{L}-\gamma_{c} \mathcal{I}\right) v=\mu_{c} v, \quad \mu_{c}=\left(\lambda-\gamma_{c}\right) /\left(\lambda-\sigma_{c}\right) .
$$

It was found that the real shift-invert option was the most efficient for our problem. Since the bifurcation from the waves is of Hopf type $\sigma_{s i}$ was taken positive, and of the order of the imaginary part of the right-most eigenvalues, which was determined initially, in the parameter region of interest, with the first method. The first 12 eigenvalues of maximal modulus of the transformed system were computed to ensure that the relevant eigenvalues were not missed. For this purpose the subroutines DENAUPD and DENEUPD of the ARPACK package [40] were used. The right position of the bifurcation points was checked with the first method. Since computing the eigenvalues requires the action of the transformed operator, systems with matrices of the form $\left(\mathcal{L}-\sigma_{*} \mathcal{I}\right)$ must be solved. The same solvers and preconditioning techniques used to find the waves were used. 


\section{RESULTS}

\section{A. Stability of the conduction state}

To start the continuation of the waves, initial conditions are required. They can be found by time evolution, forcing the required azimuthal invariance, or by studying the stability of the trivial state. Figure 2 shows the marginal stability curves of the conduction state for azimuthal wave numbers $m=$ $1, \ldots, 8$, and the corresponding critical frequencies. Details on their calculation and more results for a large range of parameters at low Ekman number can be found in [9]. This primary bifurcation is of Hopf type giving rise to azimuthal traveling waves due to the $\mathrm{SO}(2)$ symmetry group in the longitude. The reflection symmetry with respect to the equator is maintained in most cases, although there are parameter regions, not considered here, for which it is also broken at the first branching point [31]. Each intersection of the stability curves corresponds to a double-Hopf bifurcation. The bottom plots show the detail in the region studied. At $\eta_{c}=$ 0.33079417 , and $R_{c}=200690.45$ there is an intersection of the $m=5$ and $m=6$ curves. This point was computed with the precision shown by taking the appropriate truncation parameters. The branches of azimuthal waves close to this double Hopf have been studied in the next section.

\section{B. Branches of waves}

Figure 3 shows the branches of azimuthal waves for $m=3, \ldots, 8$, and $\eta=0.32,0.33,0.34$, and 0.35 . The $L^{2}$ norm of the solutions is represented against the Rayleigh number. The horizontal line, $\|u\|_{L^{2}}=0$, corresponds to the conduction state. As usual, solid and dashed lines indicate stable and unstable solutions, respectively. The curves corresponding to $m=1,2$ were also computed, but since they appear at higher values of $R$ (see Fig. 2) we have focused on the interval shown, where the secondary bifurcations take place. The drifting frequency $\omega$ along the branches of solutions is plotted in Fig. 4, and enlarged plots for $\eta=0.35$ can be seen in Fig. 5 .

To start each curve, the critical eigenfunction obtained by the linear stability analysis of the conduction state, scaled by a small factor, was used as the initial condition together with the critical value of the parameter $p_{c}=R_{c}^{0}$ slightly increased. The eigenfunction was also used as the reference solution $u_{c}$, which appears in the phase condition (9). The order in which the branches start when $R$ increases is given in each plot. It allows one to check the presence of the double-Hopf bifurcations, which appear when $\eta$ is moved.

The results shown in Fig. 3 are in agreement with the behavior in subregion I of the simple case of the doubleHopf bifurcation. See, for instance, [41], where a complete description of the parametric portraits and of the bifurcation diagrams close to the double-Hopf are given. When $\eta$ is increased a single parameter is moved in the parametric space, but since we also have the dependence of the curves with $R$, it is easy to see all possible scenarios. For instance, at $\eta=0.32$, and below the first bifurcation, the only solution is the conduction state which is stable. If $R$ is increased a first Hopf bifurcation occurs giving rise to a branch of stable azimuthal traveling waves of wave number $m=5$. By further increasing $R$ a second Hopf bifurcation of the conduction state gives rise to the $m=6$ unstable branch of waves, which recovers stability at a Neimark-Sacker bifurcation if $\eta$ is increased close to $\eta=0.33$.
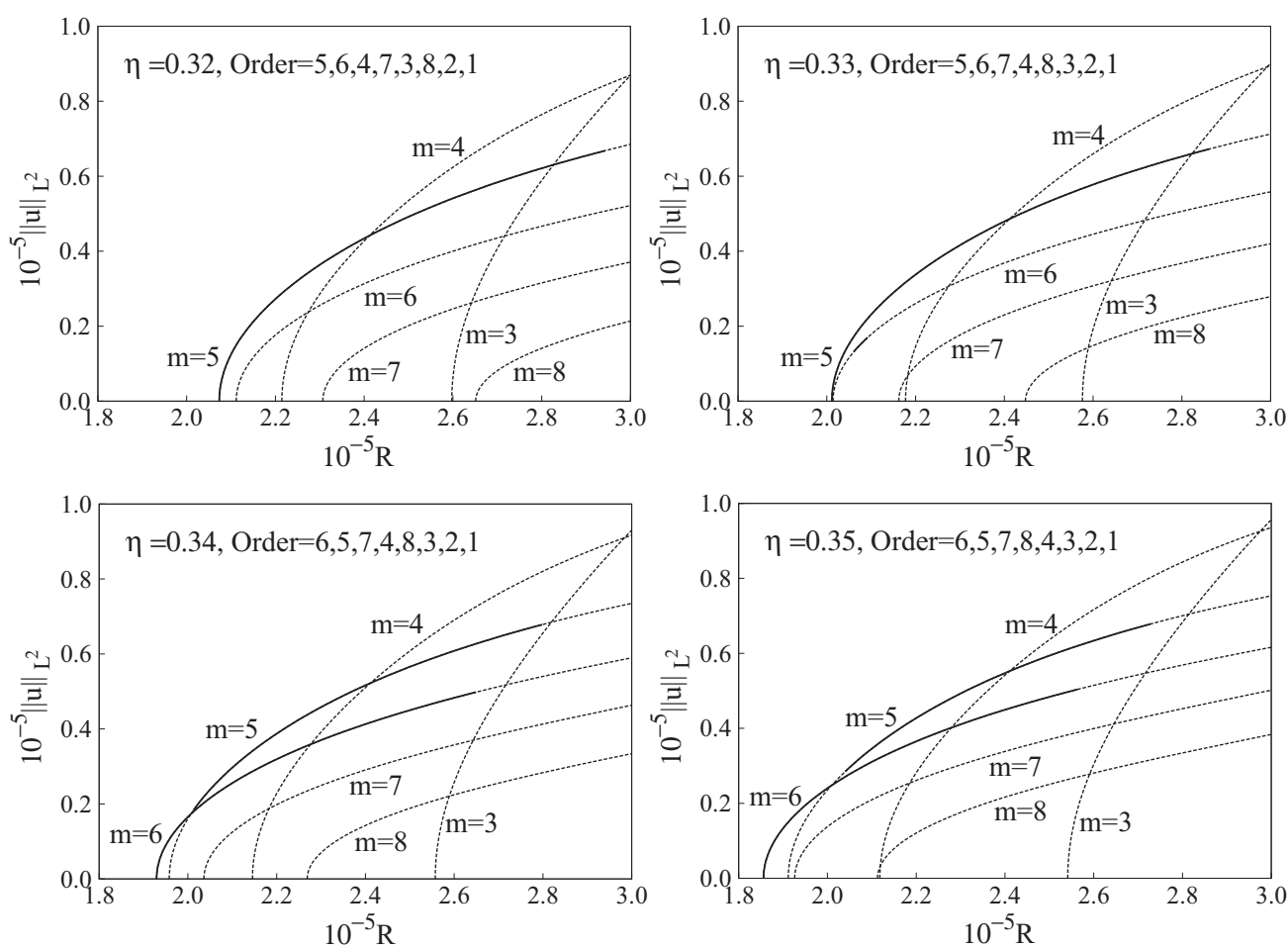

FIG. 3. Bifurcation diagrams close to the double-Hopf bifurcation point for several values of $\eta$. The label gives the order in which the branches of waves bifurcate from the conduction state when $R$ increases. 

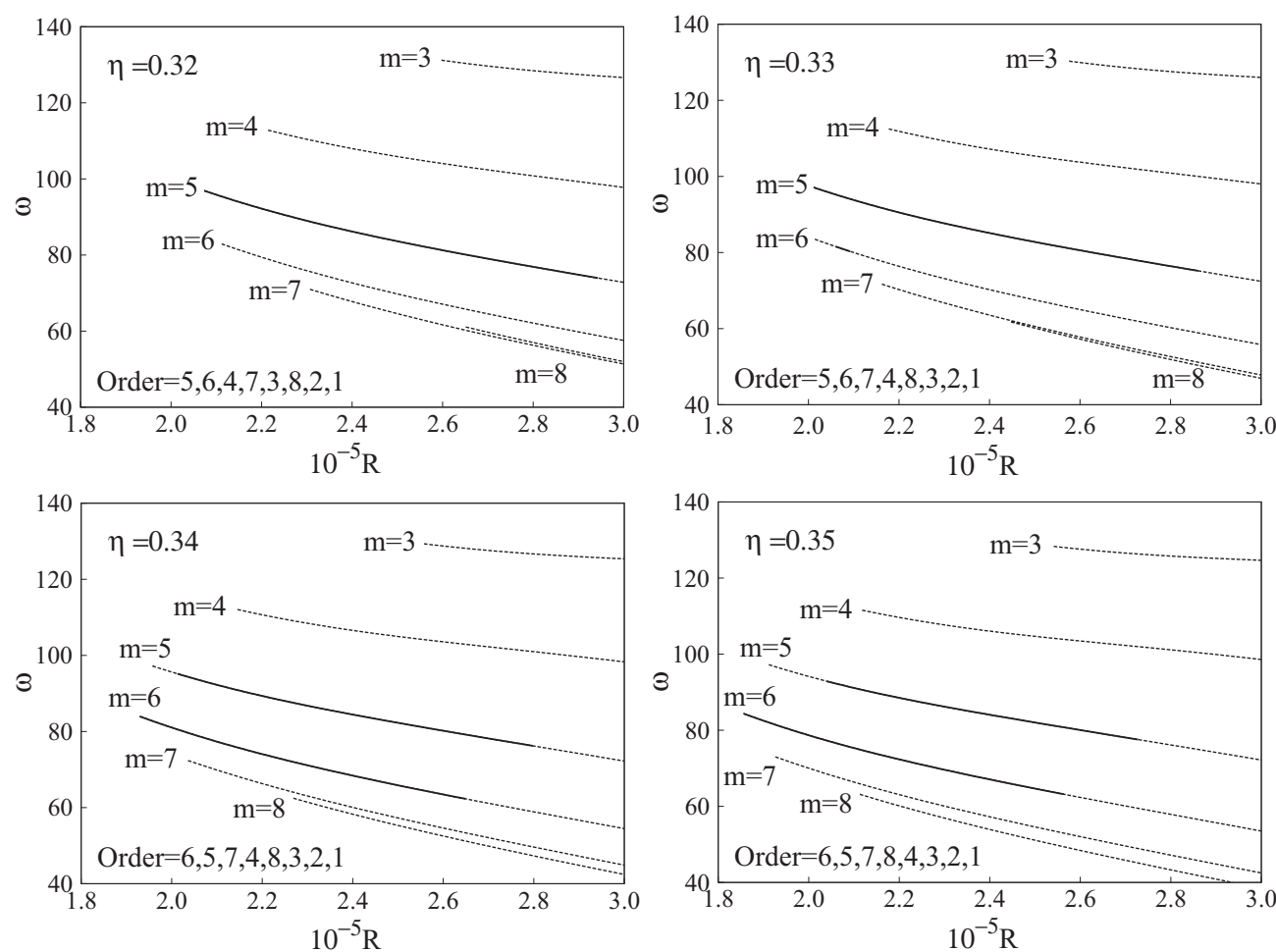

FIG. 4. Drifting frequencies along the branches of waves of Fig. 3.

A branch of unstable invariant tori (shape modulated waves) is born there. Notice the small portion of stable solutions on the
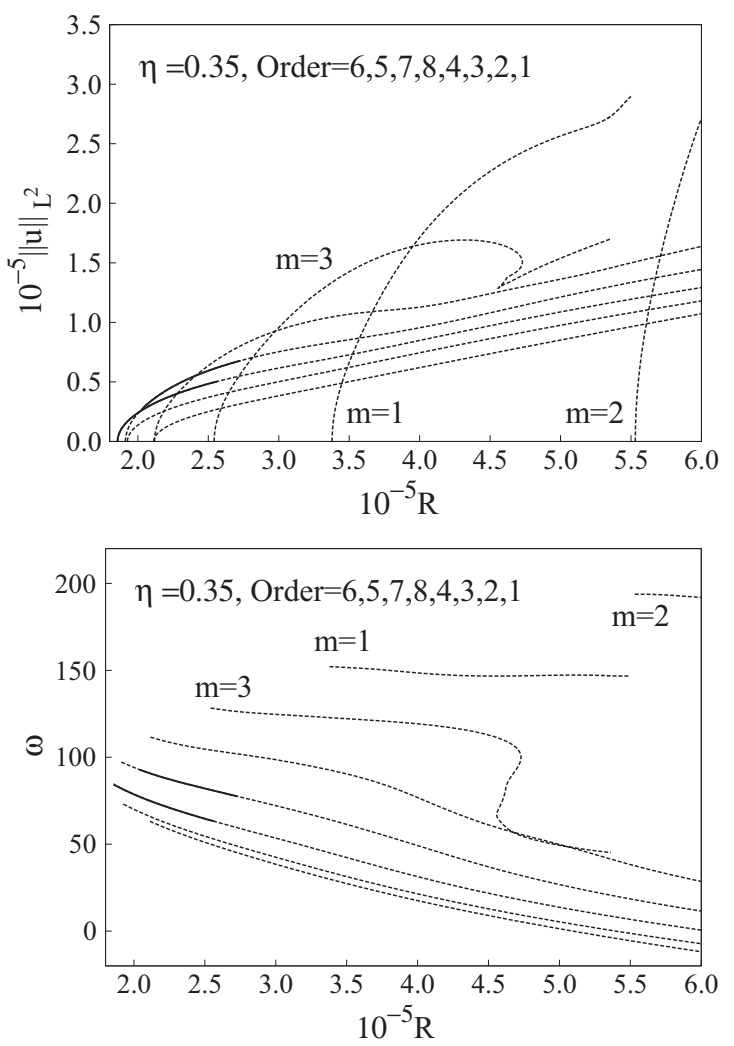

FIG. 5. Enlarged bifurcation and frequency diagrams for $\eta=$ 0.35 . The nonlabeled curves can be identified by looking at the $\eta=0.35$ plot in Figs. 3 and 4 . $m=6$ curve. By increasing $\eta$ above the double Hopf value ( $\eta_{c}=0.33079417$ and $R_{c}=200690.45$ ) the order of the bifurcations to waves of $m=5$ and $m=6$ reverses and the same sequence of events can be followed, but with the role of the two azimuthal wave numbers interchanged. Therefore the six possible phase portraits of subregion I of the simple case of the double-Hopf are present close to this codimension-two singularity. Since we can follow the waves at large distances from it, we can also observe their loss of stability. The drifting frequency is a decreasing function of $R$ in the range shown. If the product $m \omega$ is represented instead of $\omega$ the curves get closer, but have different slopes.

In Fig. 5 the curves labeled $m=1$ and $m=3$ end before reaching $R=6 \times 10^{5}$, because at these points the preconditioner starts to fail and GMRES stagnates, decreasing the residual very slowly. Since these branches did not play any role in what we were interested in describing we did not try to follow them by another method based on time integration, explained in the Conclusions section of [22]. It is, in general, more computationally expensive but very robust, and can also be used for unstable solutions. The figure also shows a fold along the $m=3$ branch, which was traced without problems with the pseudoarclength method. The structure of the solutions does not change significantly between or after the folds.

Figure 6 shows the evolution of the solutions along the $m=$ 6 branch for $\eta=0.35$. The contour levels of the perturbation of the temperature $\Theta$ are displayed on spherical, equatorial, and longitudinal sections in each row. The sections pass through a local maximum of $\Theta$, and their positions are indicated in the other two plots of the same row by a line. This also holds for the rest of the figures of the same kind. The two upper solutions are stable, and the lower two are unstable. The Neimark-Sacker 

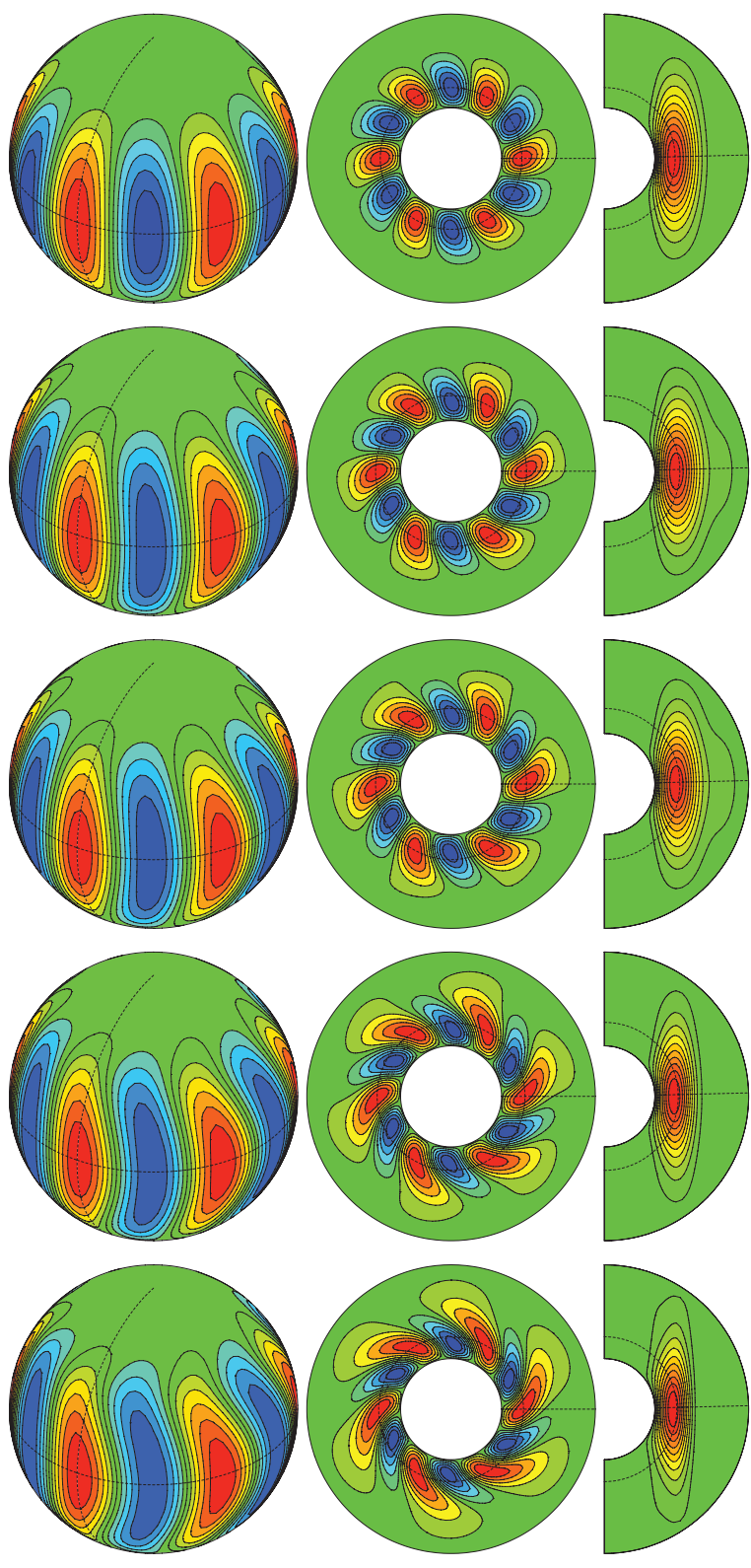

FIG. 6. (Color online) Solutions on the $m=6$ branch for $\eta=$ 0.35 and, from top to bottom, $R=1.86 \times 10^{5}, 2.27 \times 10^{5}, 2.584 \times$ $10^{5}, 3.92 \times 10^{5}$, and $6.00 \times 10^{5}$.

bifurcation takes place at $R_{c}=2.584 \times 10^{5}$ (third row). This branch was extended up to $R=6 \times 10^{5}$ to see how its increase influences the solutions. The corresponding bifurcation and frequency diagrams can be seen in Fig. 5. The most remarkable features are the enlargement and the spiraling of the convection cells in the prograde direction, and the relative radial shift of the center of the positive and negative cells, which evolve to different sizes (see the equatorial and spherical sections in Fig. 6).

We have found that the points at which the waves lose stability when $R$ increases are Neimark-Sacker bifurcations giving rise to invariant tori (amplitude modulated waves), already described, among others, in [16,19]. In agreement with these authors we have checked with a time evolution code that the quasiperiodic solutions emerging at the upper
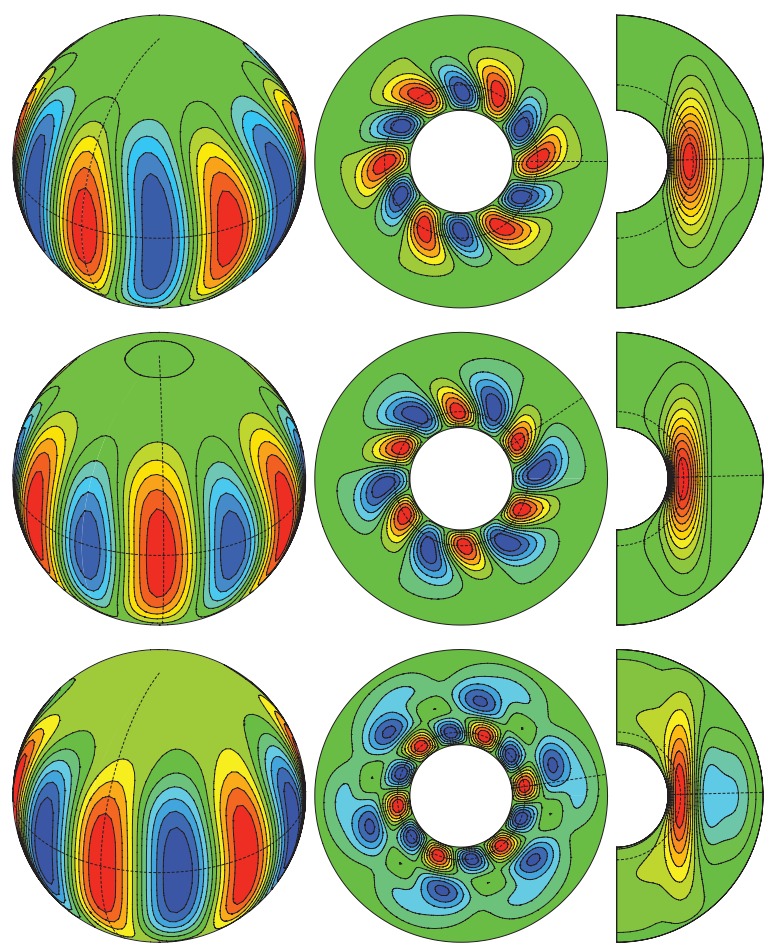

FIG. 7. (Color online) Solution at the bifurcation point on the $m=6$ branch for $\eta=0.35$ at $R=2.584 \times 10^{5}$ (top row), and corresponding real (middle) and imaginary (lower) parts of the eigenfunction. The symmetry of the solution is not broken.

bifurcations of the $m=5$ and $m=6$ stable branches are supercritical (because of their small modulation), and also stable. Those appearing at the lower bifurcations, closer to the conduction state, are unstable and break the $m$-azimuthal symmetry. Table I shows the positions of these secondary bifurcations for $\eta=0.35$, and their dependence on the number of radial points $n_{r}$ and the number of terms in the azimuthal trigonometric expansion $L / m_{d}$. In the next two columns, $n_{\text {wave }}$ means the size of the systems required to obtain the waves, and $n_{\text {stab }}$ is the size of the eigenvalue problems to study their stability. If the bifurcation does not break the azimuthal symmetry $n_{\text {stab }}=n_{\text {wave }}$, as happens on the upper bifurcations (see Figs. 7 and 8). When it is broken, all the spherical harmonics are required and $n_{\text {stab }}>n_{\text {wave }}$, since 5 and 6 are coprime (see Fig. 9). As can be seen, the maximal dimensions used to obtain the solutions of the table was 342615 to compute the waves, and 968799 to study the stability. The determination of the critical point was performed in each case by inverse interpolation. Close to a bifurcation, a polynomial $R=P(\operatorname{Re}(\lambda))$ was fitted to a set of pairs of values $\left(\operatorname{Re}\left(\lambda_{\mathrm{i}}\right), \mathrm{R}_{\mathrm{i}}\right)$, $\lambda_{i}$ being the rightmost eigenvalue for the solution of Rayleigh number $R_{i}$. The polynomial was then evaluated at $\operatorname{Re}(\lambda)=0$ to give $R_{c} \approx P(0)$. The last two columns show the drifting frequency of the waves, and the imaginary part of the rightmost eigenvalue at the bifurcation, which gives the frequency of modulation of the waves at onset. These frequencies were obtained as $R_{c}$, and have been checked by Fourier analysis of time quasiperiodic sequences near the three points [42]. In the upper bifurcation for $m=6$ and $R=2.58 \times 10^{5}$ we have found $\omega=62.80$ and $\omega^{\prime}=\operatorname{Im}(\lambda)=171.88$, and for $m=5$ 

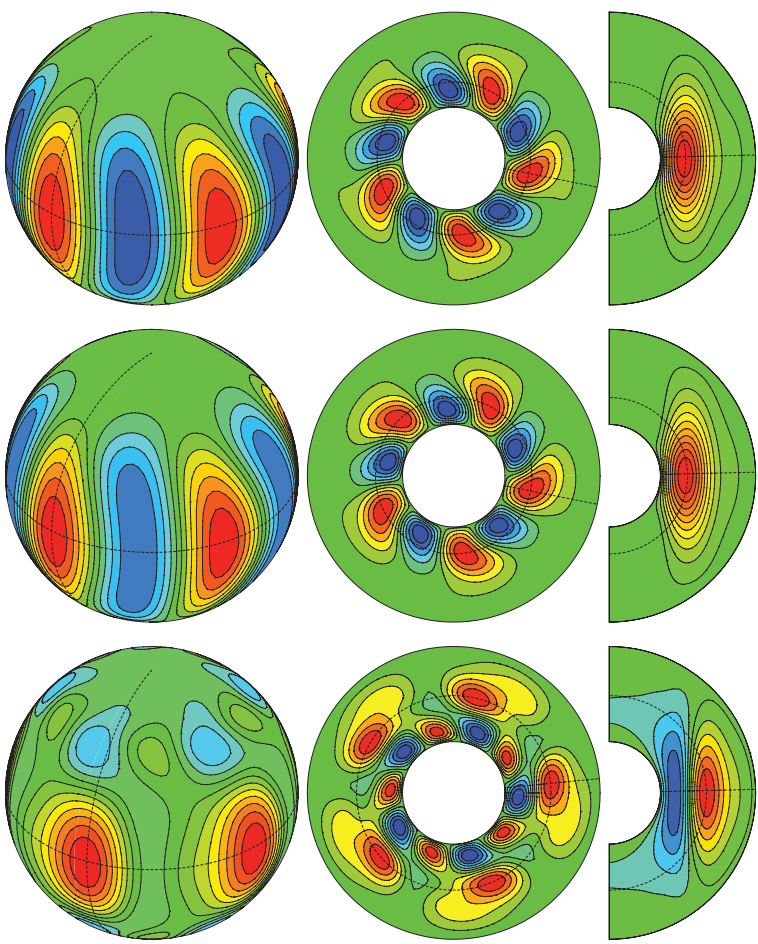

FIG. 8. (Color online) Solution at the bifurcation point on the $m=5$ branch for $\eta=0.35$ at $R=2.74 \times 10^{5}$ (top row), and corresponding real (middle) and imaginary (lower) parts of the eigenfunction. The symmetry of the solution is not broken.

and $R=2.75 \times 10^{5}, \omega=77.62$ and $\omega^{\prime}=\operatorname{Im}(\lambda)=176.98$, in very good accordance with the stability study.
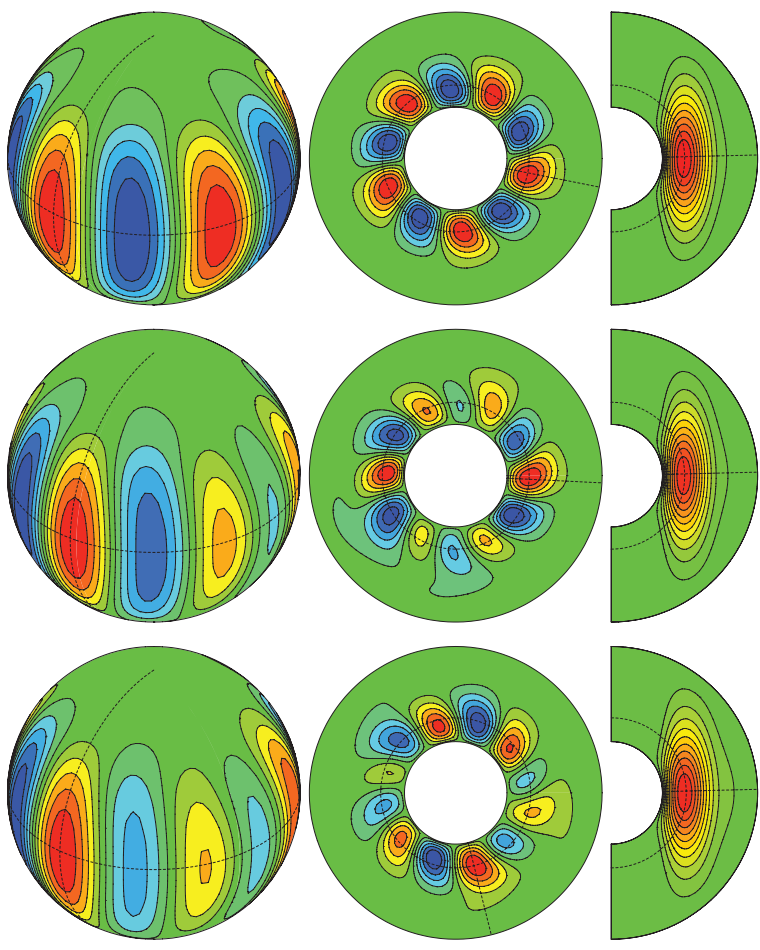

FIG. 9. (Color online) Solution at the first bifurcation point on the $m=5$ branch for $\eta=0.35$ at $R=2.043 \times 10^{5}$ (top row), and corresponding real (middle) and imaginary (lower) parts of the eigenfunction. The symmetry of the solution is broken.
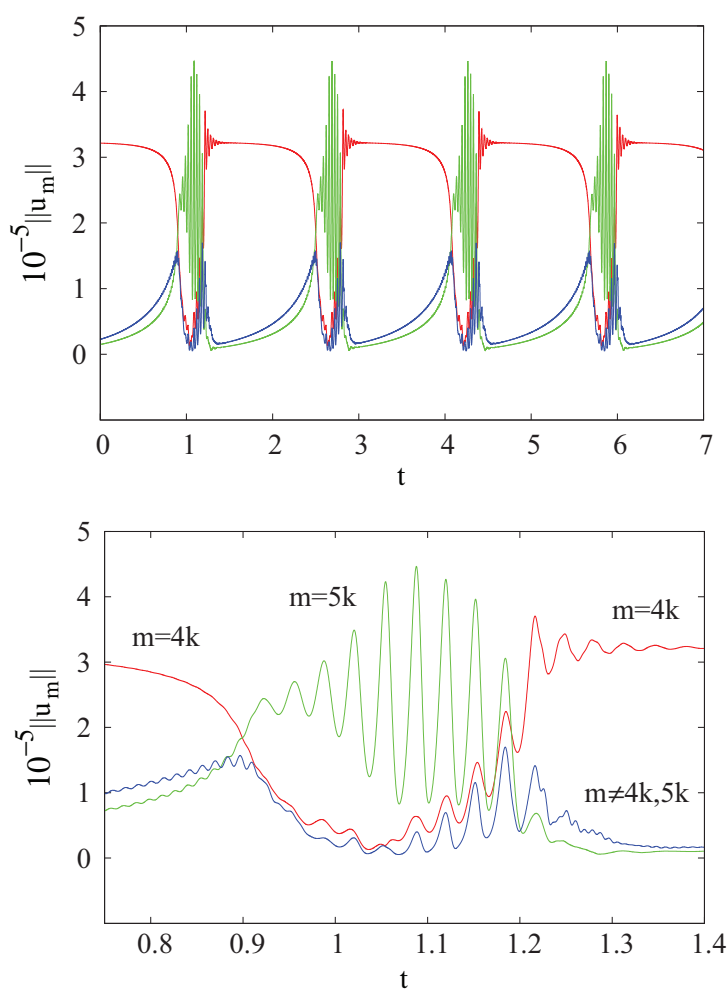

FIG. 10. (Color online) Trajectory close to a heteroclinic chain at $R=3.025 \times 10^{5}$, and a detail close to $t=1$. Norm of the amplitudes of the potentials and the temperature perturbation versus time for $m=4 k, m=5 k$, and $m \neq 4 k, 5 k$.

The different cases in the table were computed not only to check the accuracy, but also to test the robustness method when the size of the problem is increased. For the values of the parameters used here, $n_{r}=35$ and $L / m_{d}=14$ is enough to have five significant digits in $R_{c}, \omega$ and $\operatorname{Im}(\lambda)$, but at lower values of $E$ the resolution has to be increased.

Other crossings of the curves can be seen in Fig. 3, when $\eta$ is moved, between wave numbers 3-8, 4-7, and 4-8 (5-7 has not yet been reached when $\eta=0.35$ ). Therefore, in addition to the unstable periodic waves shown, there are unstable invariant tori, bifurcated from the branches of waves, and, possibly, strange repelors, emerging at bifurcations from the unstable tori. All of them can be relevant for the global dynamics of the system and in the transition to weak turbulence [43]. Orbits close to heteroclinic chains, confirming the existence of global behaviors involving these unstable objects, have been detected. An example of a nontrivial dynamics can be seen in Fig. 10. It was obtained, for $\eta=0.35$ and $R=3.025 \times 10^{5}$, by starting a time evolution for the full system of equations with an unstable $m=4$ wave as initial condition. The Euclidean norm of the amplitudes of the potentials and the temperature perturbation is plotted versus time for orders multiple of $4(m=4 k), 5$ ( $m=5 k$ ), and $m \neq 4 k, 5 k$ after an initial transient (not shown in the figure). For a pure azimuthal wave the graph of the time evolution of the norm is a constant line, because the time evolution is just a solid body rotation. After a Neimark-Sacker bifurcation, the graph of the norm for the modulated waves is periodic in time. In the plots two clearly different regimes can be seen. When the trajectory is close to an unstable pure $m=4$ 
wave, the corresponding curve is almost constant and the other two are close to zero. The latter tend to zero when the trajectory approaches the unstable wave, and grow exponentially along the unstable manifold of the periodic orbit. When the system approaches an unstable pure $m=5$ tori, the corresponding curve oscillates for a short time (see the detail in the lower plot), and the two other norms are small. This interpretation has been confirmed by looking at the evolution of contour plots, and by comparing the frequencies of these solutions with those of the unstable periodic or quasiperiodic orbits the trajectory approaches. The latter can be computed by time evolution, fixing $m_{d}=5$. In the transitions between the two regimes all the modes are nonzero. The time between visits to the periodic orbit is quite regular. We have found that, by increasing $R$, it becomes chaotic.

\section{DISCUSSION AND CONCLUSIONS}

We have described a systematic and efficient way of computing the azimuthal waves, and to study their stability in convection between corotating spheres. The time evolution codes in spherical geometry of other authors [7,44-47] could be easily modified for this purpose. The resolutions reached in the tests of accuracy, up to $n_{r}=50$ radial points and maximal degree and order of the spherical harmonics $L=90$, are possible due to the use of matrix-free iterative methods. Although it was not strictly necessary for the values of $E$ and $\sigma$ used here, they allow decreasing even more the Ekman number (or increasing the Taylor number) and increasing $m$, compared with the use of direct methods. The limit is given mainly by the amount of memory required for the preconditioner. In [46] a study of this subject for the magnetoconvection equations in spherical geometry was done, but for the block structure of the full Jacobian of the system to solve Newton's equations by direct methods. With iterative methods only part of the Jacobian has to be stored as preconditioner. Here, all the linear terms have been used. In case the storage or the time needed to perform the LU decompositions become too large, some part of them could be employed, for instance, the diagonal blocks. The only requirement is that the preconditioner used ensures the convergence of the iterative methods. Several examples in the case of the linear solvers used during the time integration by semi-implicit methods were given in [48]. An alternative based on time integrations, suggested in [22], does not require preconditioners.

There seems to be no difference in the convergence of the iterative methods if the computation of the nonlinear terms is or not de-aliased. The time required to compute each branch of periodic orbits was of the order of one day of CPU on a personal computer (Intel I7 processor at $3.20 \mathrm{GHz}$ ) depending on the length of the branch, the azimuthal wave number, the number of solutions computed due to the control of the arclength stepsize, etc. The computation of the stability was performed at a second stage. It was only done every several solutions along a given branch, and a refinement was applied when bifurcations were detected to obtain them with more precision. No parallelism was implemented although a simple use of OpenMP directives would improve the performance, as we have checked in the case of the time evolution codes.

The solutions close to a double-Hopf bifurcation were computed. The phase portraits found correspond to that of one of the regions in parameter space of the simple case. One of the consequences is that stable azimuthal traveling waves of different $m$ can coexist in certain regions of parameters. Since their intervals of stability are, generically, different, there is a range of $R$ at which one of them or both will be modulated after secondary bifurcations. This situation is very similar to that described in [49] for infinite Prandtl number and internal heating. When, by time evolution, the Rayleigh number is slowly increased from the conduction state, only one of the two paths can be observed.

Once one of the critical points at which the waves become unstable has been found, the curve of Neimark-Sacker bifurcations passing through it, for instance in the $R-E$ plane, could be followed as the locus of Hopf bifurcations of fixed points in the rotating frame of the wave. The tools needed are almost the same already used here (see [50] for a nice large-scale example). The system would be of three times the size required to compute the waves, and the most simple preconditioner would imply solving three linear systems with the same block-tridiagonal matrix.

The modulated traveling waves can also be followed by continuation methods. The basic algorithm to compute periodic orbits [22], using Newton-Krylov methods, can be applied in the frame of reference at which the quasiperiodic modulation becomes a periodic orbit. This method, but using direct linear solvers, was used, for instance, in [51] for the plane Poiseuille flow (see [43] for more examples). In this case the size of the systems to solve is the same as for the calculation of the waves plus one unit. Since time integrations are required the computational cost of each evaluation of the function will be high, but its number and that of the actions of the Jacobian will be very small. Moreover, no preconditioner is required in this case (see also [22]).

These ideas could also be used to study other problems with symmetry groups having $\mathrm{SO}(2)$ or $\mathrm{O}(2)$ as subgroups, like thermal convection in cylinders for pure or binary mixtures [52, 53], the vortex breakdown problem [54], the corotating TaylorCouette problem for which the bifurcations from the steady axisymmetric Taylor vortices to periodic behavior have already been studied [55], convection in annular domains [56], etc.

\section{ACKNOWLEDGMENTS}

This research has been supported by Spain Ministerio de Ciencia e Innovación, and Generalitat de Catalunya under Projects No. MTM2010-16930 and No. 2009-SGR-67, respectively.
[1] S. Chandrasekhar, Hydrodynamic and Hydromagnetic Stability (Oxford University Press, New York, 1961).

[2] P. H. Roberts, Astrophys. J. 141, 240 (1965).
[3] F. H. Busse, J. Fluid Mech. 44, 441 (1970).

[4] C. A. Jones, A. M. Soward, and A. I. Mussa, J. Fluid Mech. 405, 157 (2000). 
[5] E. Dormy, A. M. Soward, C. A. Jones, D. Jault, and P. Cardin, J. Fluid Mech. 501, 43 (2004).

[6] A. P. Bassom, A. M. Soward, and S. V. Starchenko, J. Fluid Mech. 689, 376 (2011).

[7] G. Glatzmaier, J. Comput. Phys. 55, 461 (1984).

[8] K. Zhang and F. H. Busse, Geophys. Astrophys. Fluid Dyn. 39, 119 (1987).

[9] M. Net, F. Garcia, and J. Sánchez, J. Fluid Mech. 601, 317 (2008).

[10] M. Net, F. Garcia, and J. Sánchez, Phys. Fluids 24, 064101 (2012).

[11] M. Ardes, F. H. Busse, and J. Wicht, Phys. Earth Planet. Inter. 99, 55 (1997).

[12] A. Tilgner and F. Busse, J. Fluid Mech. 332, 359 (1997).

[13] F. Al-Shamali, M. Heimpel, and J. Aurnou, Geophys. Astrophys. Fluid Dyn. 98, 153 (2004).

[14] P. Cardin and P. Olson, Phys. Earth Planet. Inter. 82, 235 (1994).

[15] U. Christensen, J. Fluid Mech. 470, 115 (2002).

[16] R. Simitev and F. H. Busse, New J. Phys. 5, 97.1 (2003).

[17] J. Aurnou and M. Heimpel, Icarus 169, 492 (2004).

[18] K. Zhang, Phys. Earth Planet. Inter. 68, 156 (1991).

[19] K. Zhang, Phys. Earth Planet. Inter. 72, 236 (1992).

[20] K. Kimura, S.-I. Takehiro, and M. Yamada, Phys. Fluids 23, 074101 (2011).

[21] J. Sánchez, F. Garcia, and M. Net, in Proceedings of the 7th European Nonlinear Dynamics Conference (ENOC 2011), edited by G. Bernardini, D. Rega and F. Romeo (electronic format, 2011).

[22] J. Sánchez, M. Net, B. García-Archilla, and C. Simó, J. Comput. Phys. 201, 13 (2004).

[23] D. Viswanath, J. Fluid Mech. 580, 339 (2007).

[24] Y. Duguet, C. C. T. Pringle, and R. R. Kerswell, Phys. Fluids 20, 114102 (2008).

[25] J. Sánchez and M. Net, Int. J. Bifurcation Chaos 20, 1 (2010).

[26] D. Puigjaner, J. Herrero, C. Simó, and F. Giralt, Physica D 240, 920 (2011).

[27] J. Sánchez, M. Net, and C. Simó, Physica D 239, 123 (2010).

[28] L. van Veen, G. Kawahara, and M. Atsushi, SIAM J. Sci. Comput. 33, 25 (2011).

[29] R. A. Secco and H. H. Schloessin, J. Geophys. Res. B 94, 5887 (1989).

[30] G. A. d. Wijs, G. Kresse, L. Vočadlo, D. Dobson, D. Alfè, M. J. Gillan, and G. D. Price, Nature (London) 392, 805 (1998).

[31] F. Garcia, J. Sánchez, and M. Net, Phys. Rev. Lett. 101, 194501 (2008).

[32] E. Knobloch, in Lectures on Solar and Planetary Dynamos, Publications of the Newton Institute, edited by M. R. E. Proctor and A. D. Gilbert (Cambridge University Press, Cambridge, England, 1994).

[33] R. C. Whaley, A. Petitet, and J. Dongarra, Automated Empirical Optimization of Software and the ATLAS Project, Tech. Rep. UT-CS-00-448 (Netlib, Lapack working notes, 2000).

[34] K. Goto and R. A. van de Geijn, ACM Trans. Math. Softw. 34, 1 (2008).

[35] M. Frigo and S. G. Johnson, Proc. IEEE 93, 216 (2005), special issue on "Program Generation, Optimization, and Platform Adaptation".

[36] S. A. Orszag, J. Atmos. Sci. 27, 890 (1970).

[37] Y. Saad and M. H. Schultz, SIAM J. Sci. Stat. Comput. 7, 865 (1986).

[38] K. Meerbergen and D. Roose, IMA J. Numer. Anal. 16, 297 (1996).

[39] R. B. Lehoucq and A. G. Salinger, Int. J. Num. Meth. Fluids 36, 309 (2001).

[40] R. B. Lehoucq, D. C. Sorensen, and C. Yang, ARPACK User's Guide: Solution of Large-Scale Eigenvalue Problems with Implicitly Restarted Arnoldi Methods (SIAM, Philadelphia, 1998).

[41] Y. A. Kuznetsov, Elements of Applied Bifurcation Theory, Second Edition (Springer, New York, 1998).

[42] F. Garcia Gonzalez, Thermal Convection in Rotating Spherical Shells, Ph.D. thesis, Universitat Politècnica de Catalunya, 2012.

[43] G. Kawahara, M. Uhlmann, and L. van Veen, Annu. Rev. Fluid Mech. 44, 203 (2012).

[44] T. L. Clune, J. R. Elliot, M. S. Miesch, J. Toomre, and G. A. Glatzmaier, Parallel Comput. 25, 361 (1999).

[45] A. Tilgner, Int. J. Numer. Methods Fluids 30, 713 (1999).

[46] R. Hollerbach, Int. J. Numer. Methods Fluids 32, 773 (2000).

[47] D. J. Ivers, ANZIAM Journal 44, C400 (2003).

[48] F. Garcia, M. Net, B. García-Archilla, and J. Sánchez, J. Comput. Phys. 229, 7997 (2010).

[49] K. Zhang, J. Fluid Mech. 228, 607 (1991).

[50] A. G. Salinger, R. B. Lehoucq, R. P. Pawlowski, and J. N. Shadid, Int. J. Numer. Methods Fluids 40, 1059 (2002).

[51] P. S. Casas and A. Jorba, Theoret. Comput. Fluid Dyn. 18, 285 (2004).

[52] M. Net, I. Mercader, and E. Knobloch, Phys. Fluids 7, 1553 (1995).

[53] J. N. Sorensen and E. A. Christensen, Phys. Fluids 7, 764 (1995).

[54] J. Sánchez, F. Marqués, and J. M. López, J. Comput. Phys. 180, 78 (2002)

[55] J. Antonijoan and J. Sánchez, Phys. Fluids 12, 3147 (2000).

[56] M. Net and J. Sánchez, Int. J. Bifurcation Chaos 15, 3953 (2005). 